\title{
Impacts of Pedestrians on Capacity and Delay of Major Street Through Traffic at Two-Way Stop-Controlled Intersections
}

\author{
Zhao Yang, ${ }^{1}$ Yuanyuan Zhang, ${ }^{2}$ Renwei Zhu, ${ }^{3}$ Xiaofei Ye, ${ }^{4}$ and Xiaohong Jiang ${ }^{5}$ \\ ${ }^{1}$ Civil Aviation College, Nanjing University of Aeronautics and Astronautics, Jiangjun Road No. 29, Nanjing 211106, China \\ ${ }^{2}$ Safe Transportation Research and Education Center, University of California, Berkeley, CA 94720, USA \\ ${ }^{3}$ China Academy of Urban Planning \& Design, Shanghai 200335, China \\ ${ }^{4}$ Maritime College, Ningbo University, Ningbo 315211, China \\ ${ }^{5}$ School of Transportation, Southeast University, Nanjing 210096, China
}

Correspondence should be addressed to Yuanyuan Zhang; yuanyuanzhang@berkeley.edu

Received 26 July 2014; Accepted 26 September 2014

Academic Editor: Huimin Niu

Copyright ( 2015 Zhao Yang et al. This is an open access article distributed under the Creative Commons Attribution License, which permits unrestricted use, distribution, and reproduction in any medium, provided the original work is properly cited.

This paper aims to estimate the impacts of pedestrians on capacity and average control delay for the major street through traffic at two-way stop-controlled (TWSC) intersections. A procedure was proposed to estimate the expected delay for major street through vehicles based on the pedestrian arrival time and motorist yielding behavior. Field data were collected to calibrate the crucial parameters in the established models. The proposed models were then validated against field measured data. It was found that the calibrated models provided reasonable delay estimates. Based on the established models, sensitivity analyses were conducted to identify the effects of pedestrian arrival rate and motorist yield rate on the capacity of the major street through lanes at TWSC intersections. The results suggested that the capacity of the through movement would decrease with an increase in the pedestrian arrival rate. It was also found that, with the same pedestrian arrival rate, the capacity would decrease as the motorist yield rate became higher, and the magnitude of the capacity reduction would increase with an increase in the pedestrian arrival rate.

\section{Background}

The current edition of the Highway Capacity Manual (HCM) proposes a procedure for estimating the capacity and delay for various traffic movements at two-way stop-controlled (TWSC) intersections. The HCM capacity estimation method assumes that a traffic movement with a lower priority yields to the right-of-way of higher order movements. Accordingly, each traffic movement at a TWSC intersection is assigned a specific ranking in a priority hierarchy. The through traffic on the major street is considered the rank 1 movement which has the absolute priority. That is, all other movements with lower ranks have to yield to the major street through traffic. Given this assumption, the capacity of a through traffic lane on the major street at a TWSC intersection is estimated as the saturation flow rate, and the delay to through vehicles is usually considered zero [1].
The major street through traffic is in conflict with the pedestrian movements crossing the major street at TWSC intersections. In the HCM, the pedestrian movement crossing the major street is defined as a rank 2 movement. Thus, the HCM method assumes that the pedestrians' crossing movement has to yield to the major street through traffic. This assumption is not so reasonable considering the fact that in many countries motorists are legally required to yield to pedestrians under most circumstances [1]. In practice, many motorists at TWSC intersections select to yield to the rightof-way of pedestrians, and the yield rate is influenced by a range of factors. A series of studies have investigated how motorists respond to pedestrians at TWSC intersections [13]. The actual motorist yield rate ranges from $17 \%$ to $91 \%$. As a result, the presence of minor street pedestrian crossing movements may have impedance effects on the capacity of the major street through traffic. 
Theoretically, the impedance effects of pedestrian movements on the capacity of major street through traffic are influenced by a number of factors, including vehicular and pedestrian flow rates, the length of a crosswalk, the critical headways for pedestrians crossing major streets, and motorist yield rates. Many studies have been conducted to estimate capacity and delay at stop-controlled intersections. The most commonly used methods include the probabilistic approach which is based on gap acceptance and major road headway distribution, as well as the deterministic approach which is based on empirical data [4-7]. Specifically, several studies have estimated the capacity of vehicular movements that are influenced by pedestrians [8-13]. HCM presented a method to calculate the average pedestrian occupancy at the conflict zone at signalized intersections when the pedestrian flow rate during the pedestrian service time was $1,000 \mathrm{p} / \mathrm{h}$ or less. The average pedestrian occupancy was then used to determine the capacity adjustment factor for the vehicular movement [1]. Viney and Pretty presented a method to estimate the saturation flow of a stream of turning vehicles at a signalized intersection when the stream was required to give way to a two-way stream of pedestrians. The green period for vehicles was divided into five intervals; in the first and third intervals, pedestrian platoons blocked the vehicles so there was no flow, in the second and fourth intervals, vehicles accepted gaps in the pedestrian stream, and in the final period the pedestrian flow had ceased so vehicles were unopposed. The capacity in a cycle could therefore be calculated and, when divided by the green time, the saturation flow was obtained [11]. A similar study was conducted by Chen et al. The capacity of right-turning movements influenced by pedestrians at signalized intersections was estimated based on the conflict-zone-occupancy approach. The results showed that pedestrians had a strong impact on the right-turn capacities at low pedestrian volumes and that the effects of additional pedestrians decrease as the pedestrian volumes increase [12].

Although these studies provide some insights into the capacity and delay estimation for the vehicular movements, they are mostly based on the assumption that motorists have absolute priority, indicating that all the pedestrians would give way to motorists or that the pedestrians have absolute priority, indicating that all the motorists would yield to pedestrians. However, the actual yielding behaviors vary under different circumstances [14-16], and the effect was not considered in the established models.

A more recent study conducted by Wei et al. developed an analytical model to estimate the expected vehicular delay as a function of the traffic volume, pedestrian volume, and the vehicle yielding rate for the through movement at TWSC intersections. However, the paper only considered the condition that pedestrians came from one side of the street. Actually, pedestrians would arrive on both sides of the street. Besides, all the parameters in the model were based on assumed or default values from HCM, and the results from the model were not tested using field measured data [17].

The primary objective of the present study was to evaluate the impacts of pedestrians on the capacity and delay of major street through traffic at TWSC intersections. More specifically, the study presented in this paper focuses on the following tasks: (1) to develop a capacity and average control delay model for major street through vehicles which take into consideration the impedance effects of pedestrians; (2) to calibrate the crucial parameters in the capacity and delay models using field data; (3) to validate the established models using field data; and (4) to estimate the impacts of crucial parameters on the capacity of the major street through movement through a sensitivity analysis.

\section{Methodology}

2.1. Basic Assumptions. Assuming that vehicles on the major street arrive randomly, the vehicle arriving pattern follows the Poisson distribution, and the vehicle headways follow the negative exponential distribution [18]. The cumulative distribution function for vehicle headways can be expressed as

$$
H(t)=1-e^{-\mu_{i} t},
$$

where $\mu_{i}$ represents the vehicle arrival rate for the $i$ th lane. The probability density function can be expressed as

$$
h(t)=\mu_{i} e^{-\mu_{i} t} .
$$

Let $\mu$ represent the vehicle arrival rate for all through lanes. It can be estimated as

$$
\mu=\mu_{1}+\mu_{2}+\cdots+\mu_{n}
$$

where $n$ represents the number of through lanes. Assuming that the minor stream drivers consider all major stream vehicles to be identical, the $n$ traffic streams can be combined into one traffic stream [19]. The corresponding probability density function can be expressed as

$$
h(t)=\mu e^{-\mu t} .
$$

For the pedestrian flow, it is also assumed that pedestrians arrive randomly on both sides of the street. For each side, the pedestrian arrival pattern follows the Poisson distribution. Thus, the probability density function for pedestrian arrival interval can be expressed as

$$
f(t)=\lambda e^{-\lambda t}
$$

where $\lambda$ represents the pedestrian arrival rate.

2.2. Capacity of Major Street Through Traffic. When the impedance effects of pedestrians are not considered, the capacity of a through lane equals the saturation flow rate. Let $h_{s}$ represent the saturation headway for the through movement (sec). When pedestrians are present at the side of the street, some motorists may stop or decelerate to give way to pedestrians. Let $C$ represent the capacity of the exclusive through lane with the impedance effects of pedestrians (pcu/hr/ln) and let $E_{c}$ represent the average control delay experienced by motorists from through lanes on the major street when the vehicle volume reaches capacity $(\mathrm{sec})$. The total lost time during each hour for motorists due to yielding to pedestrians can 
be calculated as $C \cdot E_{c}$. Thus, the following equation can be obtained:

$$
\frac{C \cdot E_{c}}{h_{s}}+C=\frac{3600}{h_{s}} .
$$

By solving this function, it can be obtained that

$$
C=\frac{3600}{h_{s}+E_{c}} .
$$

2.3. Average Control Delay for Major Street Through Traffic. To estimate the average control delay experienced by motorists at the major street through lanes, the yielding situations are discomposed into two scenarios according to vehicle headway and pedestrian critical headway. The detailed description for each scenario is illustrated as follows.

Scenario 1: Expected Vehicle Delay with a Headway Larger Than Pedestrian Critical Headway. The first scenario deals with the situation that vehicle headway is larger than pedestrian critical headway. As shown in Figure 1, the shaded line represents the location of the crosswalk. The arrows below the line represent the arrival and departure of pedestrians coming from the left side of the street and the arrows above the line represent the arrival and departure of pedestrians coming from the right side of the street. It can be noted that the first vehicle (Veh 1 ) arrives at the crosswalk at time $t_{1}$ and passes freely with no delay. If no pedestrian arrives during the following headway, the following vehicle (Veh 2) would pass the crosswalk at time $t_{2}$. However, due to the arrival of pedestrians during the headway, Veh 2 may decelerate or stop to yield to pedestrians. For this scenario, it is assumed that Veh 2 starts to make a yielding decision from the time of $t_{2}-\delta$. The reason to this assumption can be explained as follows. If the pedestrian arrives during the time interval $\left[t_{1}, t_{2}-\delta\right]$, the pedestrian will have adequate time to cross the street before the arrival of Veh 2, without having to depend on yielding motorists. In this case, Veh 2 does not need to make a yielding decision and thus incurs no delay. If the pedestrian arrives during the time interval $\left[t_{2}-\delta, t_{2}\right]$, the pedestrian has to wait for the yielding behavior of Veh 2 or wait for the next headway. Veh 2 will experience delay if it decides to yield to the pedestrian. It is also assumed that only one yielding decision is made for each vehicle.

As mentioned before, pedestrians may arrive on both sides of the street. Let $t_{a}$ and $t_{b}$ represent the time interval from $t_{2}-\delta$ to the arrival of pedestrians from the left and right sides of the street, respectively. With the assumptions that the pedestrian arrival pattern follows the Poisson distribution, the probability that a pedestrian arrives at the time $t_{2}-\delta+t_{a}$ equals the probability of having an arrival interval of $t_{a}$. It can be obtained that

$$
f\left(t_{a}\right)=\lambda_{1} e^{-\lambda_{1} t_{a}} .
$$

Similarly,

$$
f\left(t_{b}\right)=\lambda_{2} e^{-\lambda_{2} t_{b}},
$$

where $\lambda_{1}$ and $\lambda_{2}$ represent the pedestrian arrival rate on the left and right sides of the street, respectively.

Figures 1(a) to 1(c) illustrate the situation that a pedestrian coming from the left side of the street arrives during the time interval $\left[t_{2}-\delta, t_{2}\right]$. In Figure 1(a), no pedestrian arrives at the right side of the street during the time interval $\left[t_{1}, t_{2}\right]$. In Figure 1(b), a pedestrian arrives at the right side of the crosswalk during the time interval $\left[t_{1}, t_{2}-\delta\right]$. In Figure 1(c), a pedestrian arrives at the right side of the crosswalk during the time interval $\left[t_{2}-\delta, t_{2}-\delta+t_{a}\right]$. For the three cases, the probability for Veh 2 to experience delay can be estimated as

$$
P_{11}=\int_{0}^{\delta} f\left(t_{a}\right)\left[e^{-\lambda_{2} \delta}+\int_{0}^{t_{a}} f\left(t_{b}\right) d t_{b}\right] d t_{a} \cdot M \cdot \int_{\delta}^{\infty} h(t) d t
$$

where $M$ represents the motorist yield rate, which can be expressed as a constant for a specific site [1]. Accordingly, the expected delay experienced by Veh 2 in the three cases can be estimated as

$$
\begin{aligned}
E_{11}=\int_{0}^{\delta}\left(t_{a}+\rho\right) \cdot f\left(t_{a}\right) \\
\times\left[e^{-\lambda_{2} \delta}+\int_{0}^{t_{a}} f\left(t_{b}\right) d t_{b}\right] d t_{a} \cdot M \cdot \int_{\delta}^{\infty} h(t) d t,
\end{aligned}
$$

where $\rho$ represents the lost time of Veh 2, which can be estimated as the time interval from the time for departure of the pedestrian to the time at which Veh 2 passes the crosswalk.

Similarly, Figures 1(d) to 1(f) illustrate the situation that a pedestrian coming from the right side of the street arrives during the time interval $\left[t_{2}-\delta, t_{2}\right]$. In Figure $1(\mathrm{~d})$, no pedestrian arrives at the left side of the crosswalk during the time interval $\left[t_{1}, t_{2}\right]$. In Figure $1(\mathrm{e})$, a pedestrian arrives at the left side of the crosswalk during the time interval $\left[t_{1}, t_{2}-\delta\right]$. In Figure 1(f), a pedestrian arrives at the right side of the street during the time interval $\left[t_{2}-\delta, t_{2}-\delta+t_{b}\right]$. For the three cases, the probability for Veh 2 to experience delay can be estimated as

$$
P_{12}=\int_{0}^{\delta} f\left(t_{b}\right)\left[e^{-\lambda_{1} \delta}+\int_{0}^{t_{b}} f\left(t_{a}\right) d t_{a}\right] d t_{b} \cdot M \cdot \int_{\delta}^{\infty} h(t) d t .
$$

Accordingly, the expected delay experienced by Veh 2 in the three cases can be estimated as

$$
\begin{aligned}
E_{12}=\int_{0}^{\delta}\left(t_{b}+\rho\right) \cdot f\left(t_{b}\right) \\
\times\left[e^{-\lambda_{1} \delta}+\int_{0}^{t_{b}} f\left(t_{a}\right) d t_{a}\right] d t_{b} \cdot M \cdot \int_{\delta}^{\infty} h(t) d t .
\end{aligned}
$$

Scenario 2: Expected Vehicle Delay with a Headway Smaller Than Critical Headway. The second scenario illustrates the situation that vehicle headway is smaller than critical headway and this scenario is further discomposed into 6 cases based 


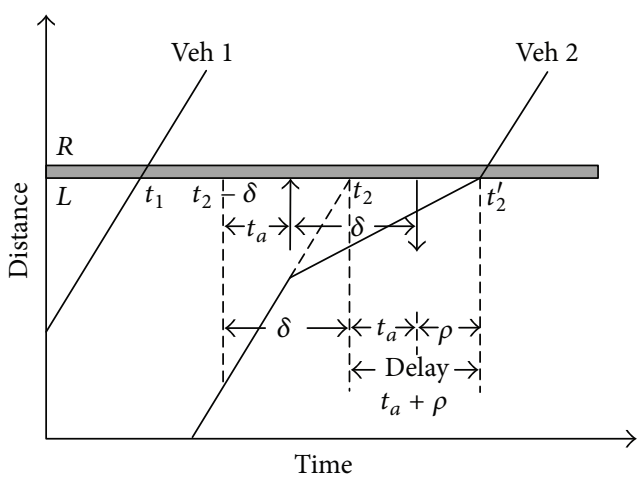

(a)

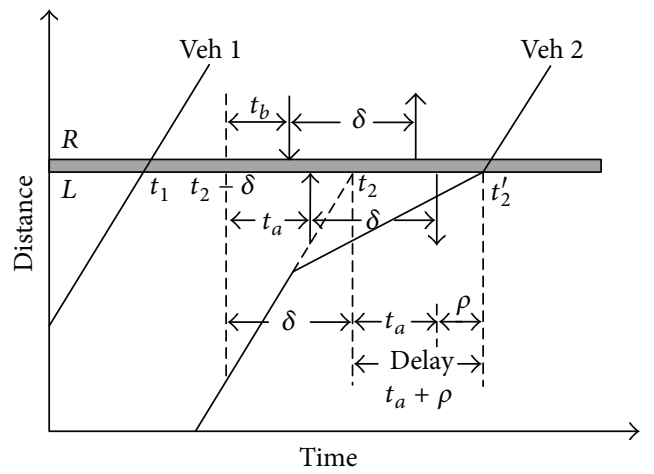

(c)

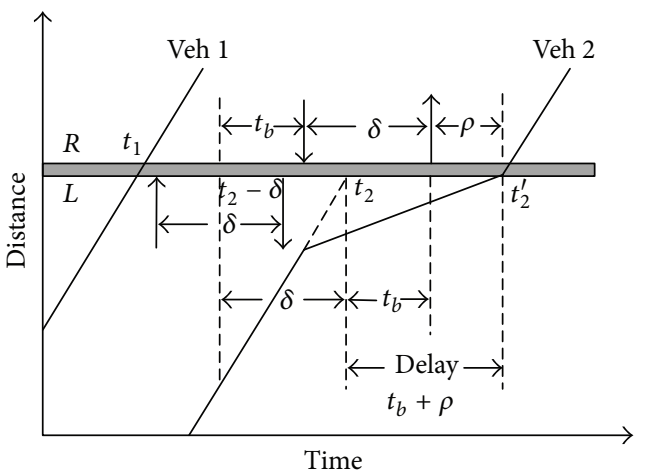

(e)

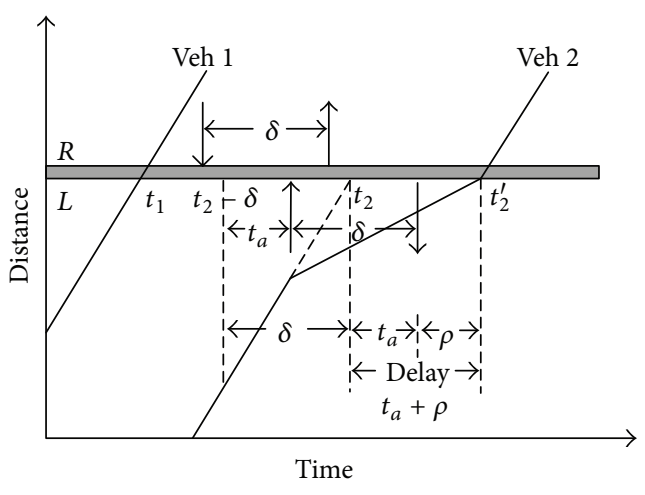

(b)

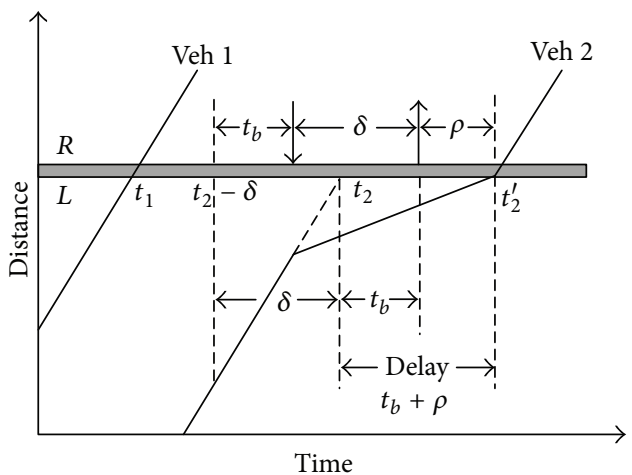

(d)

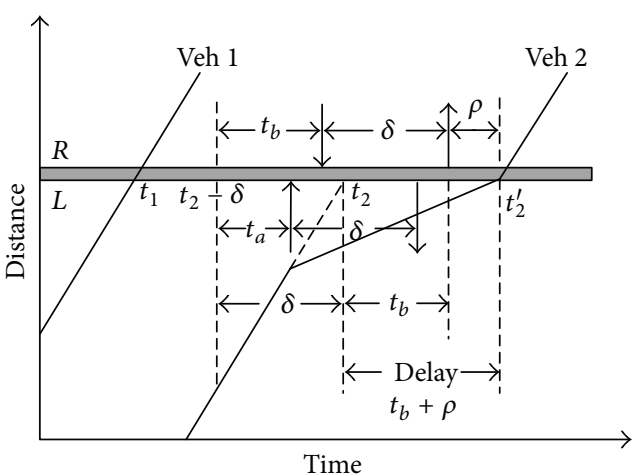

(f)

FIgURE 1: Vehicle delay for Scenario 1.

on the time of arrival for pedestrians from each side of the street, as shown in Figure 2.

The dashed arrows in the figure represent that there may be pedestrians arriving on the side of the street. Veh 1 passes the crosswalk with no delay at time $t_{1}$ and there may be pedestrians waiting on the side of the street. Veh 2 starts to make a yielding decision after the departure of Veh 1. In Figure 2(a), there are pedestrians waiting at the left side of the street at the time $t_{1}$, regardless of whether there are pedestrians waiting on the right side of the street. In Figure 2(b), there are pedestrians waiting on the right side of the street and no pedestrians waiting on the left side of the street at the time $t_{1}$. For both cases, no pedestrian arrives at either side of the street during the time interval $\left[t_{1}, t_{2}\right]$.
In this situation, Veh 1 fails to yield to the pedestrian and the pedestrian has to wait for the yielding event from the following vehicle (Veh 2) as the critical headway is smaller than vehicle headway. As Veh 2 yields to the pedestrian, the pedestrian can cross the street and Veh 2 will incur delay due to the yielding behavior. Thus, for case (a) and case (b), the probability for Veh 2 to experience delay can be calculated as

$$
\begin{aligned}
P_{21} & =[L+L(1-L)] M \int_{0}^{\delta} e^{-\lambda_{1} t} e^{-\lambda_{2} t} h(t) d t \\
& =\left(2 L-L^{2}\right) M \int_{0}^{\delta} e^{-\lambda t} h(t) d t,
\end{aligned}
$$




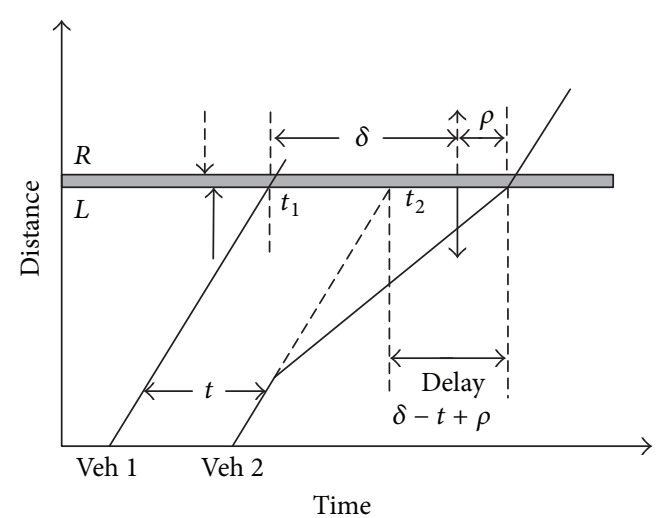

(a)

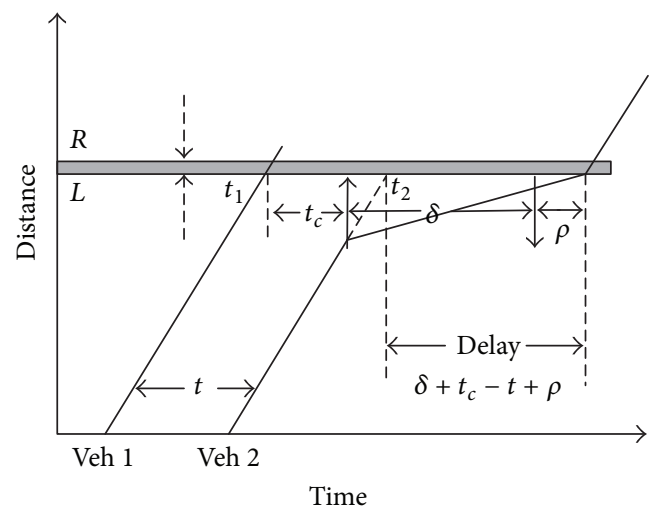

(c)

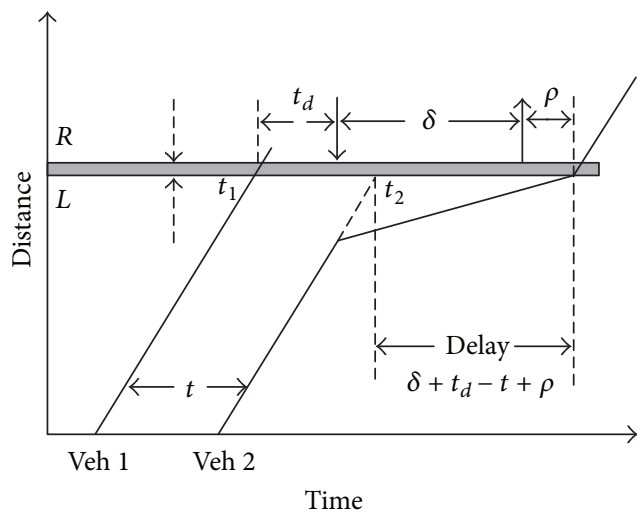

(e)

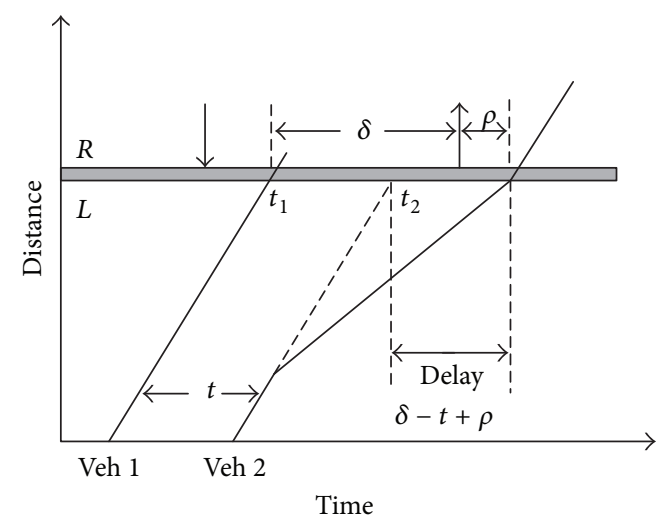

(b)

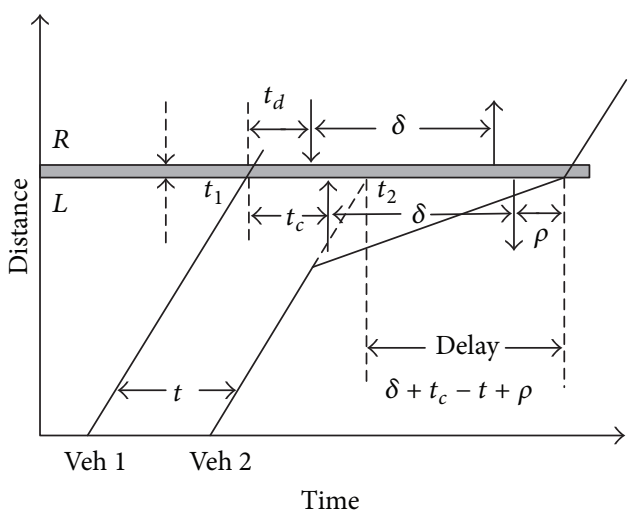

(d)

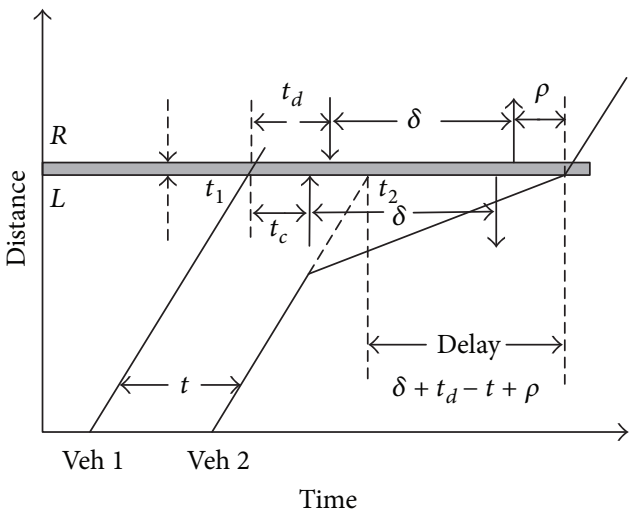

(f)

Figure 2: Vehicle delay for Scenario 2.

where $\lambda=\lambda_{1}+\lambda_{2} ; L$ represents the probability that there are pedestrians waiting to cross the street when a vehicle starts to make a yielding decision on condition that the vehicle headway is smaller than critical headway. The expected delay for Veh 2 for case (a) and case (b) can be estimated as

$$
E_{21}=\left(2 L-L^{2}\right) M \int_{0}^{\delta}(\delta-t+\rho) e^{-\lambda t} h(t) d t .
$$

Figures 2(c) and 2(d) illustrate the situation that a pedestrian arrives at the left side of the crosswalk during the time interval $\left[t_{1}, t_{2}\right]$. Let $t_{c}$ and $t_{d}$ represent the time interval from the departure of Veh $1\left(t_{1}\right)$ to the arrival of pedestrians from the left and the right sides of the street, respectively. In Figure 2(c), no pedestrian from the right arrives during the time interval $\left[t_{1}, t_{2}\right]$, while in Figure $2(\mathrm{~d})$, a pedestrian from the right arrives during the time interval $\left[t_{1}, t_{1}+t_{c}\right]$. The probability for the two cases can be calculated as

$$
P_{22}=M \int_{0}^{\delta} h(t) \int_{0}^{t} f\left(t_{c}\right)\left[e^{-\lambda_{2} t}+\int_{0}^{t_{c}} f\left(t_{d}\right) d t_{d}\right] d t_{c} d t .
$$


The expected delay for Veh 2 can be calculated as

$$
\begin{aligned}
E_{22}=M \int_{0}^{\delta} h(t) \int_{0}^{t} f\left(t_{c}\right)\left(\delta+t_{c}-t+\rho\right) \\
\times\left[e^{-\lambda_{2} t}+\int_{0}^{t_{c}} f\left(t_{d}\right) d t_{d}\right] d t_{c} d t .
\end{aligned}
$$

Similarly, Figures 2(e) and 2(f) illustrate the situation that a pedestrian arrives at the right side of the crosswalk during the time interval $\left[t_{1}, t_{2}\right]$. In Figure $2(\mathrm{e})$, no pedestrian from the right arrives during the time interval $\left[t_{1}, t_{2}\right]$. In Figure 2(f), a pedestrian from the left arrives during the time interval $\left[t_{1}, t_{1}+t_{d}\right]$. The probability for the two cases can be calculated as

$$
P_{23}=M \int_{0}^{\delta} h(t) \int_{0}^{t} f\left(t_{d}\right)\left[e^{-\lambda_{1} t}+\int_{0}^{t_{d}} f\left(t_{c}\right) d t_{c}\right] d t_{d} d t
$$

The expected delay for Veh 2 can be calculated as

$$
\begin{aligned}
E_{23}=M \int_{0}^{\delta} h(t) \int_{0}^{t} f\left(t_{d}\right)\left(\delta+t_{d}-t+\rho\right) \\
\times\left[e^{-\lambda_{1} t}+\int_{0}^{t_{d}} f\left(t_{c}\right) d t_{c}\right] d t_{d} d t .
\end{aligned}
$$

The next step is to estimate the unknown parameter $L$ in the established models. The method used here follows that in the previous research [17]. A one-step forward interaction is carried out to calculate the probability that there are pedestrians waiting on one side of the road when Veh 1 arrives at the crosswalk.

If the headway of Veh 1 is larger than the critical headway, the probability that there are pedestrians waiting when Veh 1 arrives at the crosswalk can be calculated as

$$
L_{1}=Q_{2}+(1-M) q_{1}
$$

where $Q_{2}$ represents the probability that there are more than two pedestrians arriving within the critical headway on condition that vehicle headway is larger than the critical headway. With the assumption that the vehicle only yields once, this would leave pedestrians waiting on the side of the crosswalk; $q_{1}$ represents the probability that there is one pedestrian arriving within the critical headway on condition that vehicle headway is larger than the critical headway.

If the headway of Veh 1 is smaller than the critical headway, the probability of having pedestrian waiting can be estimated as

$$
\begin{aligned}
L_{2}= & L\left[M \Phi_{1}+(1-M) H(\delta)\right] \\
& +(1-L)\left[(1-M) \varphi_{1}+\left(\Phi_{1}-\varphi_{1}\right)\right],
\end{aligned}
$$

where $\Phi_{1}$ represents the probability that there is at least one pedestrian arriving within the headway on condition that vehicle headway is smaller than the critical headway; $\varphi_{1}$ represents the probability that there is one pedestrian arriving within the headway on condition that vehicle headway is smaller than the critical headway. The first term indicates the situation that there are pedestrians waiting when Veh 1 starts to make a yielding decision. The second term indicates the situation that there is no pedestrian waiting when Veh 1 starts to make a yielding decision, but at least one pedestrian is left from the departure of the leading vehicle to the arrival of Veh 1 , as

$$
L=L_{1}+L_{2} \text {. }
$$

By solving this function, it can be obtained that

$$
L=\frac{(1-M)\left(q_{1}+\varphi_{1}\right)+\Phi_{2}+Q_{2}}{1-M \varphi_{1}-(1-M) \varphi_{0}}
$$

where $\Phi_{2}$ represents the probability that there are more than two pedestrians arriving within the headway on condition that vehicle headway is smaller than the critical headway; $\varphi_{0}$ represents the probability that no pedestrian arrives within the headway on condition that vehicle headway is smaller than the critical headway.

With the Poisson arrival of the pedestrian flow, it can be obtained that

$$
\begin{gathered}
q_{0}=[1-H(\delta)] e^{-\lambda \delta}=e^{-\beta \delta} \\
q_{1}=[1-H(\delta)] \lambda \delta e^{-\lambda \delta}=\lambda \delta e^{-\beta \delta} \\
\varphi_{0}=\int_{0}^{\delta} h(t) e^{-\lambda t} d t=\frac{\mu}{\beta}\left(1-e^{-\beta \delta}\right) \\
\varphi_{1}=\int_{0}^{\delta} h(t) \lambda t e^{-\lambda t} d t=\frac{\lambda \mu}{\beta^{2}}\left[1-e^{-\beta \delta}(\beta \delta+1)\right],
\end{gathered}
$$

where

$$
\beta=\lambda+\mu \text {. }
$$

Therefore, $Q_{2}$ and $\Phi_{2}$ can be calculated as

$$
\begin{aligned}
& Q_{2}=1-H(\delta)-q_{0}-q_{1}=e^{-\mu \delta}-q_{0}-q_{1}, \\
& \Phi_{2}=H(\delta)-\varphi_{0}-\varphi_{1}=1-e^{-\mu \delta}-\varphi_{0}-\varphi_{1} .
\end{aligned}
$$

Based on the above calculation, the probability that a vehicle is experiencing delay can be estimated as

$$
P=P_{11}+P_{12}+P_{21}+P_{22}+P_{23} \text {. }
$$

The expected vehicle delay can be estimated as

$$
E=E_{11}+E_{12}+E_{21}+E_{22}+E_{23} .
$$

The expected vehicle delay to delayed vehicles can be estimated as

$$
E_{d}=\frac{E}{P} .
$$

Based on these equations, it can be found that the average expected delay for major street through vehicles is determined by the following parameters: pedestrian arrival rate $\left(\lambda_{1}, \lambda_{2}\right)$, vehicle arrival rate $(\mu)$, pedestrian critical headway $(\delta)$, lost time $(\rho)$, and motorist yield rate $(M)$. The pedestrian critical headway, lost time, and motorist yield rate can be estimated as constant values for a specific site. Therefore, the average delay is generally determined by the vehicle and pedestrian arrival rates. 
TABLE 1: Selected sites for field data collection.

\begin{tabular}{|c|c|c|c|c|c|c|c|c|}
\hline \multirow{2}{*}{ Site } & \multirow{2}{*}{ Intersection } & \multirow{2}{*}{ Approach } & \multicolumn{3}{|c|}{ Major street configuration } & \multirow{2}{*}{$\begin{array}{c}\text { Minor street configuration } \\
\text { LTR }^{\mathrm{d}}\end{array}$} & \multirow{2}{*}{$\begin{array}{l}\text { Signs and } \\
\text { markings }\end{array}$} & \multirow{2}{*}{$\begin{array}{l}\text { Median refuge } \\
\text { island }^{\mathrm{e}}\end{array}$} \\
\hline & & & $\mathrm{L}^{\mathrm{a}}$ & $\mathrm{T}^{\mathrm{b}}$ & $\mathrm{TR}^{\mathrm{c}}$ & & & \\
\hline 1 & Shattuck Ave. \& Virginia St. & NB & 1 & 1 & 1 & 1 & High visibility & $\mathrm{Y}$ \\
\hline 2 & Shattuck Ave. \& Berkeley Way & NB & 1 & 1 & 1 & 1 & Standard & $\mathrm{Y}$ \\
\hline 3 & $\begin{array}{l}\text { Martin Luther King Jr. Way \& } \\
\text { Addison St. }\end{array}$ & SB & 1 & 1 & 1 & 1 & Continental & $\mathrm{N}$ \\
\hline 4 & San Pablo Ave. \& Hearst Ave. & NB & 1 & 1 & 1 & 1 & Standard & $\mathrm{Y}$ \\
\hline 5 & San Pablo Ave. \& Jones St. & SB & 1 & 1 & 1 & 1 & Standard & $\mathrm{Y}$ \\
\hline 6 & San Pablo Ave. \& Camelia St. & SB & 1 & 1 & 1 & 1 & Standard & $\mathrm{Y}$ \\
\hline
\end{tabular}

${ }^{a} \mathrm{~L}$ represents the exclusive left-turn lane.

${ }^{\mathrm{b}} \mathrm{T}$ represents the exclusive through lane.

${ }^{\mathrm{c}} \mathrm{TR}$ represents the shared through and right-turn lane.

${ }^{d}$ LTR represents the shared left-turn, through, and right-turn lane.

${ }^{\mathrm{e}}$ Median refuge island represents whether median refuge island is installed in the selected site (Y: yes; N: no).

\section{Data Collection}

To calibrate and validate the established models, the average vehicle delay and motorist yielding behaviors were observed in the field. The research team collected traffic data at 6 approaches to 6 TWSC intersections in Berkeley, CA. The following criteria were applied in the site selection process:

(1) the selected approach should have an exclusive through lane on the major street at a TWSC intersection;

(2) there were moderate numbers of pedestrians crossing major streets;

(3) there were limited numbers of bicyclists;

(4) lane widths should be at least 9 feet;

(5) the approach grade was level;

(6) the selected sites should be 250 feet away from upstream traffic signals.

Geometric characteristics of the selected sites are given in Table 1. A video camera was set up in the field for recording traffic data. The video cameras were inconspicuously mounted to avoid altering pedestrian or motorist behaviors. Field data collection was conducted during weekday peak periods under fine weather conditions. In total, the research team recorded 3 hours of traffic data in the field.

The recorded video tapes were later reviewed in the laboratory for obtaining traffic data. A reference line was marked in the video as the location where the delayed motorists generally started to decelerate, as shown in Figure 3. The following pieces of information were collected: (1) the vehicle volume for each movement; (2) the pedestrian volume from each side of the street; (3) the exact time at which each pedestrian started crossing the street and arrived at the other side of the street; (4) the exact time at which the rear wheel of a vehicle from the through lane crossed reference line $\mathrm{A}$ and crosswalk B; (5) whether the through vehicle is delayed or not; and (6) whether the coming vehicle yielded to pedestrians when there were pedestrians waiting for an adequate gap to cross the street. With the recoded data, the time interval for

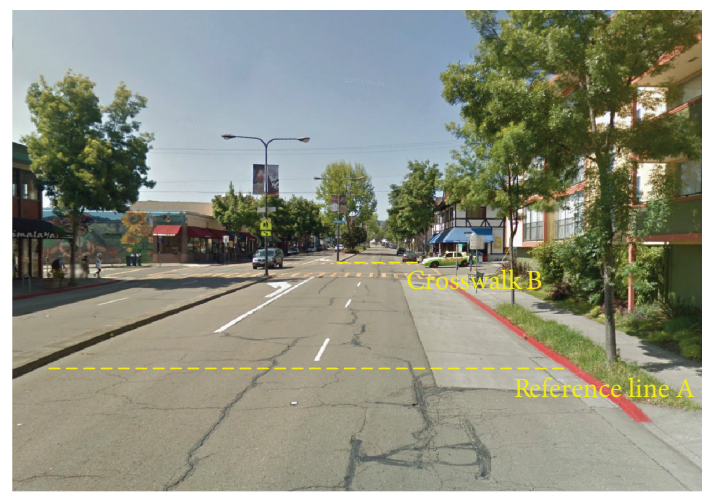

FIgURE 3: A typical site for field data collection.

each pedestrian to cross the street as well as the travel time for each through vehicle from A to B could be obtained. The average delay to delayed vehicles can be estimated as the difference in average travel time from A to B between delayed vehicles and nondelayed vehicles. It should be noted that only the motorists yielding to pedestrians are included as delayed vehicles. The vehicles which are impeded by leading delayed vehicles are excluded from this study. The lost time of each yielding event $(\rho)$ was calculated as the average time interval from the time at which the pedestrian reached the end of the crosswalk to the time when the rear wheel of the yielding vehicle passed the crosswalk (B).

For this study, the motorist yield rate was calculated as the ratio of the number of motorists who decelerated or stopped to yield to a pedestrian to the total number of motorists who should have decelerated or stopped, as shown in

$$
\begin{aligned}
& \text { Motorist yield rate (\%) } \\
& =\frac{\text { Number of motorists yielding to pedestrians }}{\text { Number of motorists who should have yielded }} .
\end{aligned}
$$

The motorist yield rate was calculated for each individual pedestrian attempting to cross the street. If there was no 
TABLE 2: Field measured data for crucial parameters and estimated delay for each site.

\begin{tabular}{|c|c|c|c|c|c|c|c|c|c|c|c|}
\hline \multirow{2}{*}{ Site } & \multirow{2}{*}{$\lambda_{1}(\mathrm{ped} / \mathrm{s})$} & \multirow{2}{*}{$\lambda_{2}(\mathrm{ped} / \mathrm{s})$} & \multirow{2}{*}{$\mu(\mathrm{pcu} / \mathrm{s})$} & \multirow{2}{*}{$t_{p}(\mathrm{sec})$} & \multirow{2}{*}{$M$} & \multicolumn{5}{|c|}{$\rho(\mathrm{sec})$} & \multirow{2}{*}{$E(\mathrm{sec})$} \\
\hline & & & & & & $N$ & Min & Max & Mean & Std. & \\
\hline 1 & 0.0139 & 0.0128 & 0.1083 & 7.54 & 0.87 & 58 & -6 & 4.88 & 0.17 & 2.89 & 6.56 \\
\hline 2 & 0.0064 & 0.0047 & 0.0917 & 6.73 & 0.65 & 27 & -4.04 & 4.48 & 1.13 & 2.31 & 6.76 \\
\hline 3 & 0.0133 & 0.0092 & 0.1556 & 12.32 & 0.79 & 43 & -8.04 & 5.60 & -1.76 & 3.72 & 8.91 \\
\hline 4 & 0.0128 & 0.0144 & 0.1139 & 6.41 & 0.72 & 41 & -4.03 & 5.92 & 1.83 & 2.91 & 8.77 \\
\hline 5 & 0.0092 & 0.0169 & 0.0753 & 8.25 & 0.83 & 54 & -2.27 & 3.01 & 0.42 & 3.09 & 9.12 \\
\hline 6 & 0.0075 & 0.0086 & 0.0839 & 9.93 & 0.92 & 22 & -3.01 & 4.99 & 1.01 & 3.94 & 11.53 \\
\hline
\end{tabular}

TABLE 3: Field measured delay and absolute percent error for each site.

\begin{tabular}{|c|c|c|c|c|c|c|c|c|c|c|c|c|}
\hline \multirow{2}{*}{ Site } & \multicolumn{5}{|c|}{$T_{d}(\mathrm{sec})$} & \multicolumn{5}{|c|}{$T_{n}(\mathrm{sec})$} & \multirow{2}{*}{$\begin{array}{c}E_{\text {Observed }}{ }^{\mathrm{a}} \\
(\mathrm{sec})\end{array}$} & \multirow{2}{*}{$\mathrm{APE}_{E}$} \\
\hline & $N$ & Min & Max & Mean & Std. dev. & $N$ & Min & Max & Mean & Std. dev. & & \\
\hline 1 & 58 & 4.44 & 17.96 & 10.30 & 3.19 & 302 & 3.24 & 7.24 & 4.56 & 0.66 & 5.74 & $14.29 \%$ \\
\hline 2 & 27 & 8.80 & 27.80 & 14.51 & 4.24 & 217 & 5.20 & 13.00 & 7.10 & 1.56 & 7.41 & $8.82 \%$ \\
\hline 3 & 43 & 9.76 & 18.12 & 12.56 & 1.99 & 165 & 3.08 & 6.24 & 4.42 & 0.58 & 8.14 & $9.46 \%$ \\
\hline
\end{tabular}

${ }^{\mathrm{a}} E_{\text {Observed }}$ represents the observed delay for delayed vehicles.

vehicle arriving at the selected site when a pedestrian crossed the street, this crossing event was not included in the calculations, as there were no motorists who should have yielded. The motorist yield rate was calculated for each site and the results were used in further analyses.

For each site, the critical headway for a pedestrian to cross the street is also estimated in the field. As there is no pedestrian platooning, the critical headway for a single pedestrian is computed using the following equation:

$$
t_{c}=t_{p}+t_{s}
$$

where $t_{c}$ represents the critical headway for a single pedestrian (sec); $t_{p}$ is the average time for pedestrians to pass the crosswalk (sec); and $t_{s}$ is the pedestrian start-up time and end clearance time $(3 \mathrm{sec})$. It should be noted that when a raised pedestrian-median refuge island is available, pedestrians typically cross in two stages [1]. Thus, $t_{p}$ is estimated as the average time for pedestrians to cross half of the crosswalk.

\section{Model Calibration and Validation}

As mentioned before, the average delay of through vehicles impeded by pedestrians is determined by the following parameters: pedestrian arrival rate $\left(\lambda_{1}, \lambda_{2}\right)$, vehicle arrival rate $(\mu)$, pedestrian critical headway $(\delta)$, lost time $(\rho)$, and motorist yield rate $(M)$. Based on the field measured data, the results of the crucial parameters are summarized in Table 2.

As shown in Table 2, the motorist yield rate was different for each site, which ranges from 0.72 to 0.92 . The means and ranges of lost time were also summarized in Table 2. The negative values indicate that the yielding vehicle passed the crosswalk before the pedestrian reaches the end of the crosswalk. For each site, the expected delay to delayed vehicles was calculated based on the proposed models using Matlab.
The estimated delay obtained from the established models was also compared to field measured results. The mean absolute percent error (MAPE) was used to measure the differences between the estimated delay and the field measured results. The MAPE value can be calculated using the following equation:

$$
\text { MAPE }=\frac{1}{N} \sum_{i=1}^{N}\left|\frac{E_{e}^{i}-E_{f}^{i}}{E_{f}^{i}}\right|,
$$

where MAPE represents the mean absolute percent error between the estimated and the field measured delay; $N$ represents the number of sites; $E_{e}^{i}$ represents the average expected delay for site $i$ which is estimated by using the established model (sec/pcu); $E_{f}^{i}$ is the field measured delay for site $i$ (sec/pcu).

The results for Site 1 to Site 3 are summarized in Table 3. As shown in the table, $T_{d}$ represents the observed travel time for delayed vehicles (sec); $T_{n}$ represents the observed travel time for nondelayed vehicles (sec); $E_{\text {Observed }}$ represents the observed average control delay (sec). $E_{\text {Observed }}$ was calculated as the difference between the travel time for delayed vehicles and that for nondelayed vehicles. The delay model developed in this study yielded a MAPE value of $10.86 \%$ as compared with the field measured delay.

The model was tested with some different sites (Site 4 to Site 6). The estimated capacity was used to validate the model, as shown in Table 4 . The capacity calculated using the proposed model $\left(C_{\text {Estimated }}\right)$ was also compared with the capacity estimated using the HCM method $\left(C_{\mathrm{HCM}}\right)[1]$, which is based on the saturation flow rate theory. The results indicated that the proposed model provided better capacity estimates for the through traffic, as compared with that used by HCM. 
TABLE 4: Comparison of estimated capacity between proposed models and traditional methods.

\begin{tabular}{|c|c|c|c|c|c|c|c|}
\hline Site & $h_{S}{ }^{\mathrm{a}}$ & $E_{\text {Observed }}^{\mathrm{b}}(\mathrm{sec})$ & $C_{\text {Observed }}(\mathrm{veh} / \mathrm{h})$ & $C_{\text {Estimated }}(\mathrm{veh} / \mathrm{h})$ & $\mathrm{APE}_{\mathrm{Cl}}{ }^{\mathrm{c}}$ & $C_{\mathrm{HCM}}(\mathrm{veh} / \mathrm{h})$ & $\mathrm{APE}_{C 2}{ }^{\mathrm{d}}$ \\
\hline 4 & 1.87 & 0.699 & 1401 & 1209 & $13.72 \%$ & 1925 & $37.38 \%$ \\
\hline 5 & 1.92 & 0.87 & 1290 & 1253 & $2.89 \%$ & 1875 & $45.31 \%$ \\
\hline 6 & 1.99 & 0.79 & 1295 & 1415 & $9.27 \%$ & 1809 & $39.70 \%$ \\
\hline
\end{tabular}

${ }^{\mathrm{a}} h_{S}$ represents the saturation headway.

${ }^{\mathrm{b}} E_{\text {Observed }}$ represents the observed delay for all vehicles.

${ }^{c} \mathrm{APE}_{\mathrm{Cl}}$ represents the APE between the estimated capacity using the proposed method and capacity calculated using the observed data.

${ }^{\mathrm{d}} \mathrm{APE}_{\mathrm{C} 2}$ represents the APE between the estimated capacity using the HCM method and capacity calculated using the observed data.

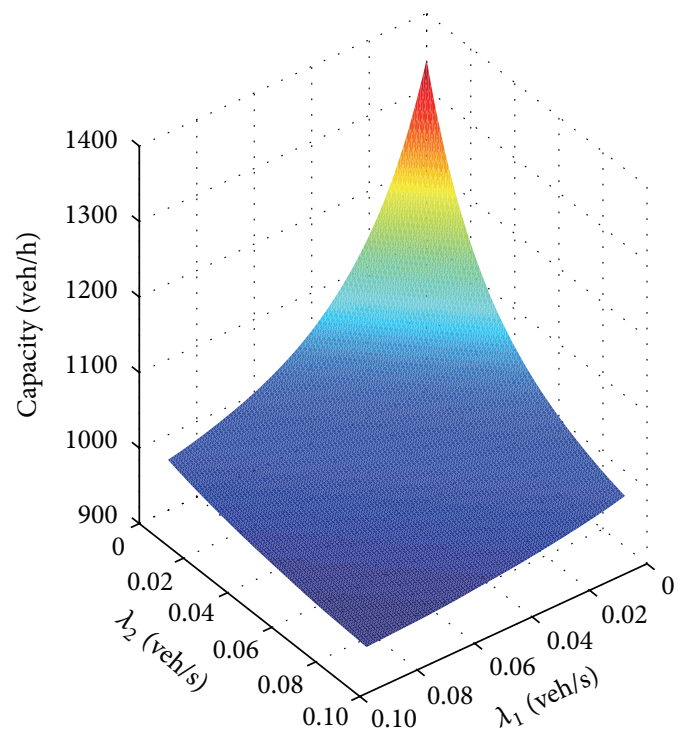

$M=0.2$

(a)

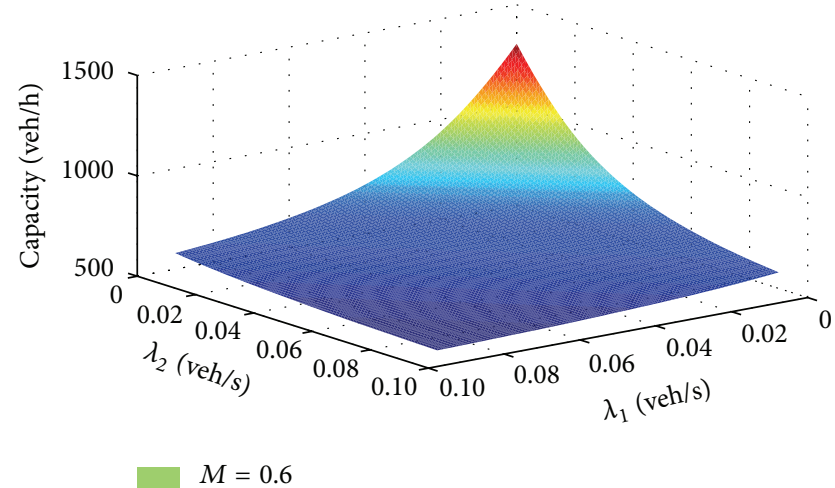

(c)

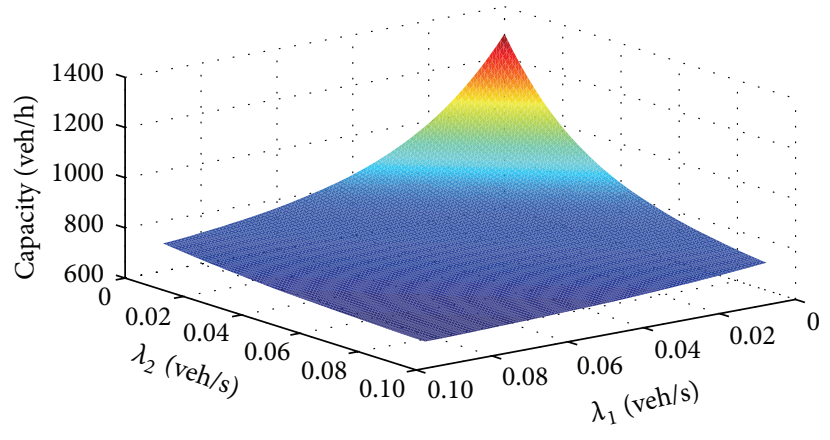

$M=0.4$

(b)

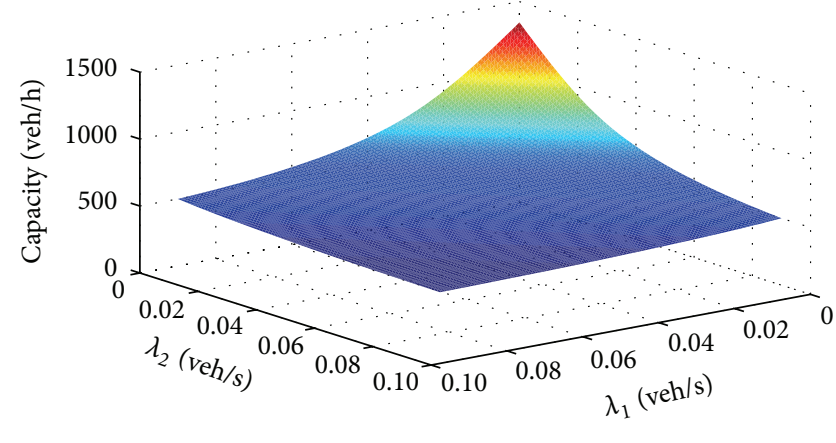

$M=0.8$

(d)

Figure 4: Comparison of capacities of an exclusive through lane with different motorist yield rates.

\section{Sensitivity Analysis}

With the established delay model, the capacity of a major street through movement can be obtained using (7). Sensitivity analyses were further conducted to identify the effects of crucial parameters on the capacity of the exclusive through lanes at TWSC intersections. The parameters considered in the sensitivity analyses include pedestrian arrival rate $\left(\lambda_{1}, \lambda_{2}\right)$ and motorist yield rate $(M)$.

Assuming that the pedestrian critical headway $(\delta)$ is $10 \mathrm{sec}$, the lost time $(\rho)$ is $2 \mathrm{sec}$, and the saturation headway $\left(h_{s}\right)$ is $2 \mathrm{sec}$, the effect of pedestrians on the capacity of major street through lanes at TWSC intersections is illustrated in Figure 4 . The curves in Figure 4 show that the capacity of 
the through movement decreases with an increase in the pedestrian arrival rate. This finding is reasonable as the more pedestrians arrive at the crosswalk, the more impendence effects will be caused to motorists. The motorist yield rate is also a factor that affects the capacity of through lanes at TWSC intersections. As shown in Figure 4, the capacities of the through movements with different motorist yield rates are generally comparable under low pedestrian volume conditions. However, with the same pedestrian arrival rate, the capacity decreases as the motorist yield rate becomes higher, and the magnitude of the capacity reduction increases with an increase in the pedestrian arrival rate.

\section{Conclusions and Discussions}

The study evaluated the impacts of pedestrians on capacity and average control delay for the major street through movement at TWSC intersections. A procedure was proposed to estimate the expected delay for major street through vehicles based on the pedestrian arrival time and motorist yielding behavior. Field data were collected to calibrate the crucial parameters in the established models, including pedestrian arrival rate $\left(\lambda_{1}, \lambda_{2}\right)$, vehicle arrival rate $(\mu)$, average time for pedestrians to cross the street $\left(t_{p}\right)$, lost time $(\rho)$, and motorist yield rate $(M)$. The proposed models were then validated against field measured data. It was found that the calibrated models provided reasonable delay estimates.

Based on the established capacity and delay models, sensitivity analyses were conducted to identify the effects of crucial parameters on the capacity of the exclusive through lanes at TWSC intersections. The results suggested that the capacity of the through movement would decrease with an increase in the pedestrian arrival rate. It was also found that, with the same pedestrian arrival rate, the capacity would decrease as the motorist yield rate became higher, and the magnitude of the capacity reduction would increase with an increase in the pedestrian arrival rate.

The capacity and delay models developed in this study can be directly used for estimating the capacity and delay for the major street through movements at TWSC intersections, which can be a supplement to the capacity estimation method for TWSC intersections provided in HCM [1]. One of the limitations in this study lies in that the expected delay estimated using the proposed model only accounts for the delay experienced by motorists who yield to the pedestrians. However, the delay experienced by the following motorists who have to decelerate due to the delayed leading vehicles was not calculated in this study. Further research needs to be conducted to obtain more accurate results. Besides, this study used a constant motorist yield rate for each site. Actually, there are many factors that would affect the motorist responses to pedestrians, such as the travel speeds and pedestrians crossing treatments. Further research is still needed to better understand those factors affecting the motorist yielding behaviors. Additional studies are also needed to test for the transferability of the capacity and delay models using data collected from other sites in other cities. The authors recommend that future studies focus on these issues.

\section{Conflict of Interests}

The authors declare that there is no conflict of interests regarding the publication of this paper.

\section{References}

[1] Highway Capacity Manual, TRB, National Research Council, Washington, DC, USA, 2010.

[2] S. Turner, K. Fitzpatrick, M. Brewer, and E. S. Park, "Motorist yielding to pedestrians at unsignalized intersections," Transportation Research Record: Journal of the Transportation Research Board, vol. 1982, pp. 1-12, 1982.

[3] H. Huang, C. Zegeer, R. Nassi, and B. Fairfax, "The effects of innovative pedestrian signs at unsignalized locations: a tale of three treatments," Tech. Rep. FHWA-RD-00-098, 2000.

[4] M. Kyte, C. Clemow, N. Mahfood, B. K. Lall, and C. J. Khisty, "Capacity and delay characteristics of two-way stop-controlled intersections," Transportation Research Record, no. 1320, pp. 160-167, 1991.

[5] M. Kyte, J. Zegeer, and B. K. Lall, "Empirical models for estimating capacity and delay at stop-controlled intersections in the United States," in Intersections without Traffic Signals II, pp. 335361, 1991.

[6] J. J. Lu and B. K. Lall, "Empirical analysis of traffic characteristics at two-way stop-controlled intersections in Alaska," Transportation Research Record, no. 1495, pp. 49-56, 1995.

[7] A. J. Khattak and P. P. Jovanis, "Capacity and delay estimation for priority unsignalized intersections: conceptual and empirical issues," Transportation Research Record, no. 1287, pp. 129-137, 1990.

[8] N. Lalani and ITE Pedestrian and Bicycle Task Force, "Alternative treatments for at-grade pedestrian crossings," Tech. Rep. LP-629, Institute of Transportation Engineers, Washington, DC, USA, 2001.

[9] C. V. Zegeer, J. R. Stewart, H. H. Huang, and P. A. Lagerwey, "Safety effects of marked versus unmarked crosswalks at uncontrolled locations: executive summary and recommended guidelines," Tech. Rep. FHWA-RD-01-075, FHWA, U.S. Department of Transportation, 2002.

[10] Y. Bian, J. Lu, and L. Zhao, "Method to determine pedestrians level of service for unsignalized intersections," Applied Mechanics and Materials, vol. 253-255, no. 1, pp. 1936-1943, 2013.

[11] N. D. Viney and R. L. Pretty, "Saturation flow of a movement subject to a pedestrian stream at traffic signals," in Proceedings of the 11th Conference of the Australian Road Research Board, pp. 157-166, 1982.

[12] X.-M. Chen, C.-F. Shao, and Y. Hao, "Influence of pedestrian traffic on capacity of right-turning movements at signalized intersections," Transportation Research Record, no. 2073, pp. 114-124, 2008.

[13] D. Helbing, R. Jiang, and M. Treiber, "Analytical investigation of oscillations in intersecting flows of pedestrian and vehicle traffic," Physical Review E: Statistical, Nonlinear, and Soft Matter Physics, vol. 72, no. 4, Article ID 046130, 2005.

[14] B. J. Schroeder and N. M. Rouphail, "Event-based modeling of driver yielding behavior at unsignalized crosswalks," Journal of Transportation Engineering, vol. 137, no. 7, pp. 455-465, 2011.

[15] B. Schroeder, A behavior-based methodology for evaluating pedestrian-vehicle interaction at crosswalks [Ph.D. thesis], North Carolina State University, Raleigh, NC, USA, 2008. 
[16] A. J. Mayne, "Some further results in the theory of pedestrians and road traffic," Biometrika, vol. 41, pp. 375-389, 1954.

[17] D. L. Wei, J. Kumfer, H. Liu, Z. Tian, and C. Yuan, "An analytical delay model to yielding vehicles at unsignalized pedestrian crossings," in Proceedings of the Transportation Research Board 92nd Annual Meeting, Washington, DC, USA, January 2013.

[18] R. J. Cowan, "Useful headway models," Transportation Research, vol. 9, no. 6, pp. 371-375, 1975.

[19] R. J. Troutbeck, "Average delay at an unsignalized intersection with two major streams each having a dichotomized headway distribution," Transportation Science, vol. 20, no. 4, pp. 272-286, 1986. 


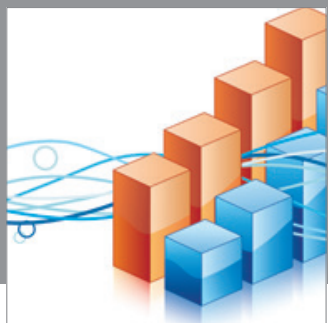

Advances in

Operations Research

mansans

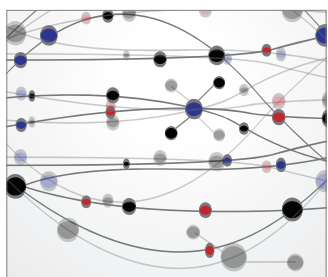

The Scientific World Journal
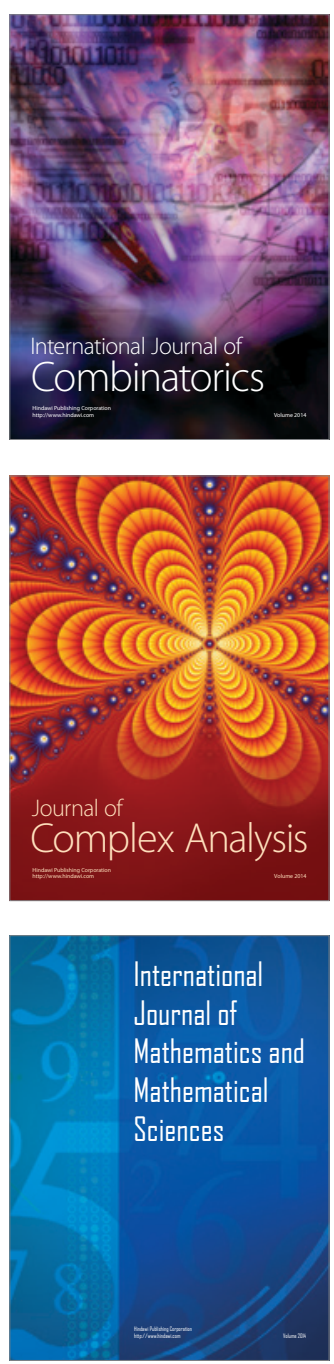
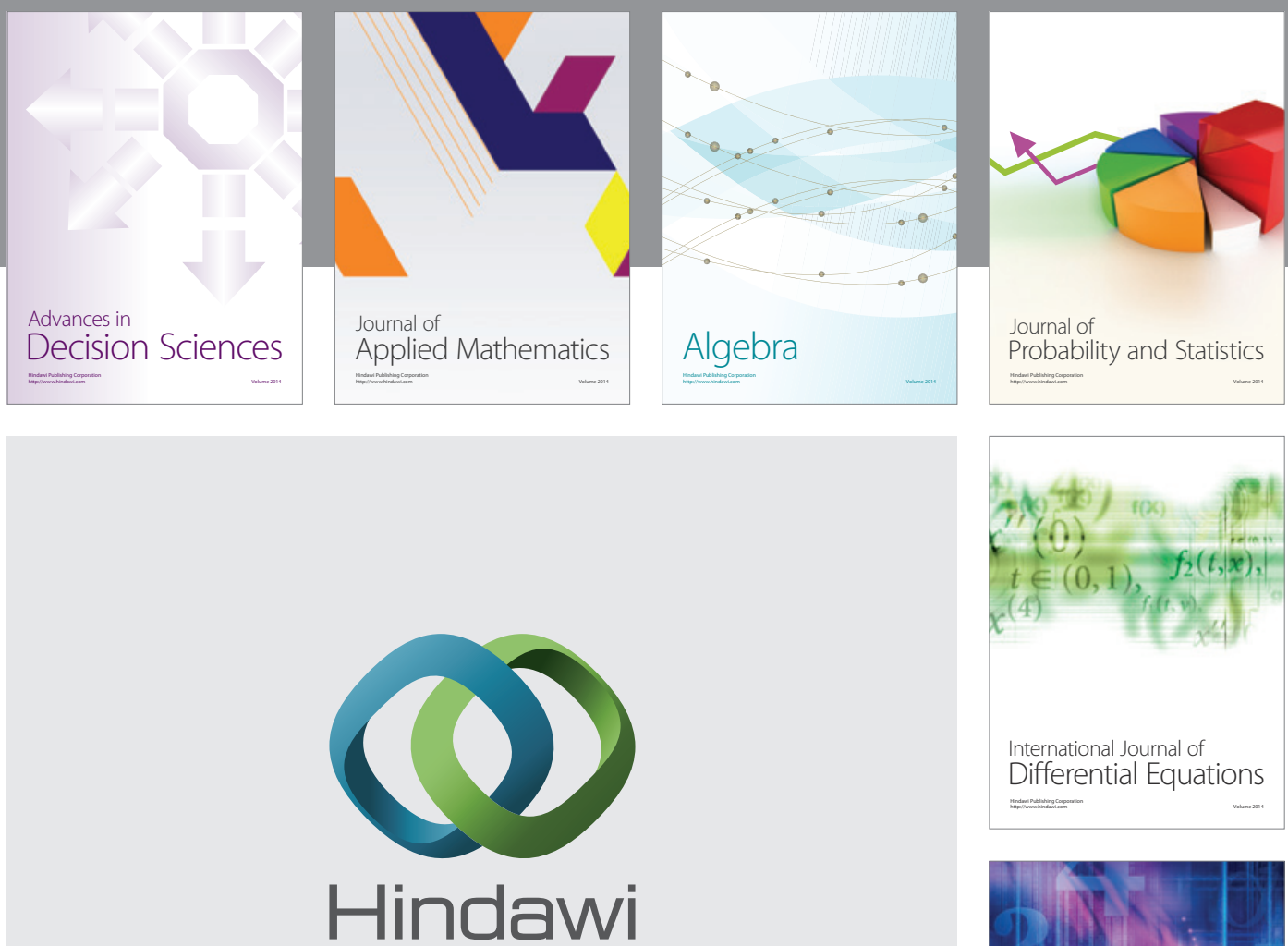

Submit your manuscripts at http://www.hindawi.com
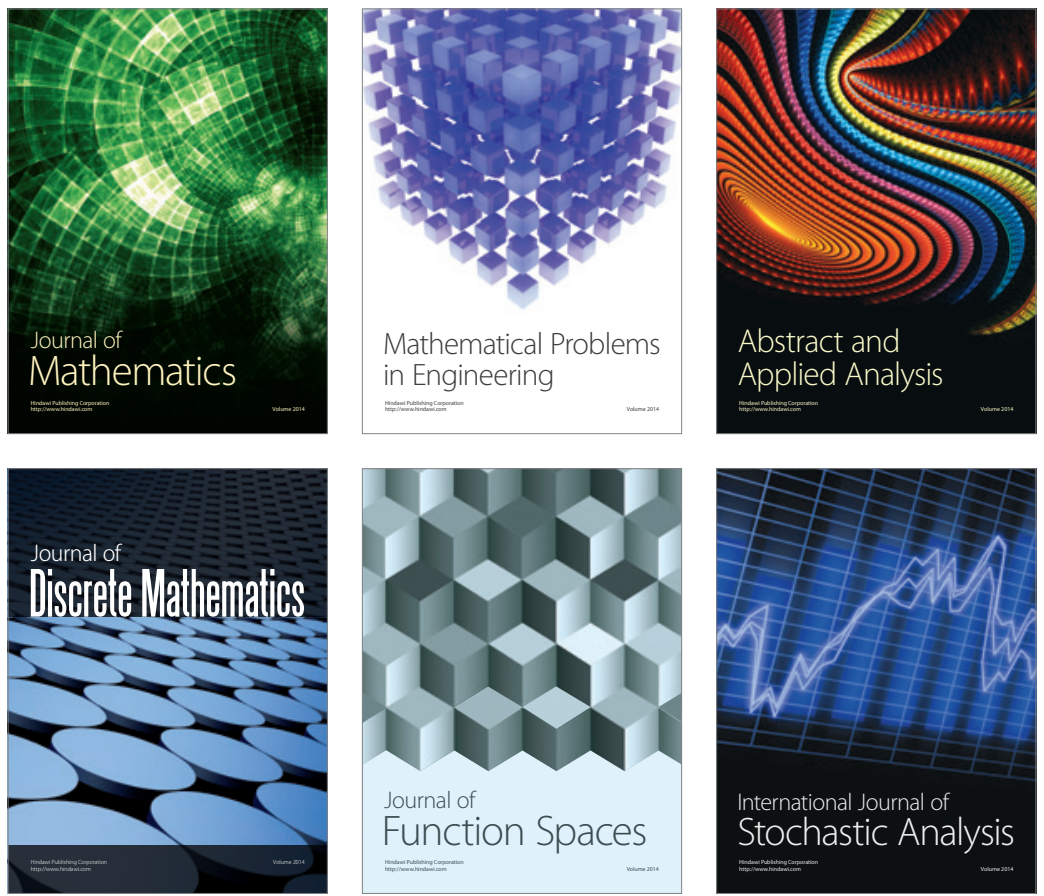

Journal of

Function Spaces

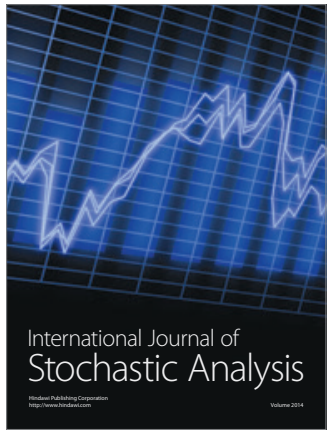

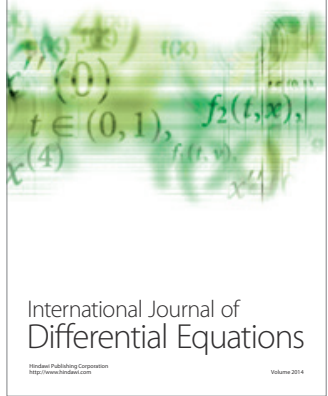
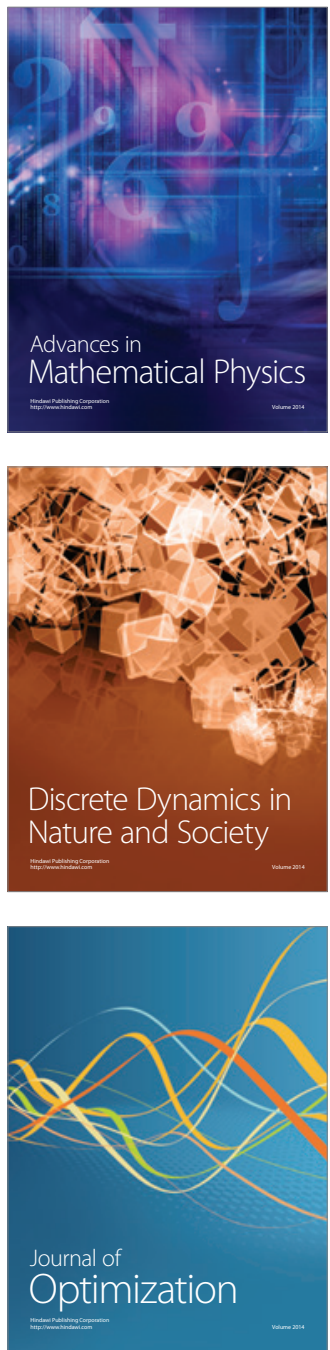